\title{
Capabilities and Limits of Compact Error Resilience Methods for Algorithmic Self-Assembly
}

\author{
Sudheer Sahu $\cdot$ John H. Reif
}

Received: 29 June 2007 / Accepted: 24 March 2008

(C) Springer Science+Business Media, LLC 2008

\begin{abstract}
Winfree's pioneering work led the foundations in the area of errorreduction in algorithmic self-assembly (Winfree and Bekbolatov in DNA Based Computers 9, LNCS, vol. 2943, pp. 126-144, 2004), but the construction resulted in increase of the size of assembly. Reif et al. (Nanotechnol. Sci. Comput. 79-103, 2006) contributed further in this area with compact error-resilient schemes that maintained the original size of the assemblies, but required certain restrictions on the Boolean functions to be used in the algorithmic self-assembly. It is a critical challenge to improve these compact error resilient schemes to incorporate arbitrary Boolean functions, and to determine how far these prior results can be extended under different degrees of restrictions on the Boolean functions. In this work we present a considerably more complete theory of compact error-resilient schemes for algorithmic selfassembly in two and three dimensions. In our error model, $\epsilon$ is defined to be the probability that there is a mismatch between the neighboring sides of two juxtaposed tiles and they still stay together in the equilibrium. This probability is independent of any other match or mismatch and hence we term this probabilistic model as the independent error model. In our model all the error analysis is performed under the assumption of kinetic equilibrium. First we consider two-dimensional algorithmic self-assembly. We present an error correction scheme for reduction of errors from $\epsilon$ to $\epsilon^{2}$ for arbitrary Boolean functions in two dimensional algorithmic self-assembly. Then we characterize the class of Boolean functions for which the error can be reduced from $\epsilon$ to $\epsilon^{3}$, and present an error correction scheme that achieves this reduction. Then we prove ultimate limits on certain classes of compact error resilient schemes: in particular we show that they can not provide reduction of errors from $\epsilon$
\end{abstract}

A preliminary version of this paper was published in DNA12 [22].

S. Sahu $(\varangle) \cdot$ J.H. Reif

Department of Computer Science, Duke University, Box 90129, Durham, NC 27708-0129, USA

e-mail: sudheer@cs.duke.edu

J.H. Reif

e-mail: reif@cs.duke.edu 
to $\epsilon^{4}$ is for any Boolean functions. Further, we develop the first provable compact error resilience schemes for three dimensional tiling self-assemblies. We also extend the work of Winfree on self-healing in two-dimensional self-assembly (Winfree in Nanotechnol. Sci. Comput. 55-78, 2006) to obtain a self-healing tile set for threedimensional self-assembly.

\section{Introduction}

\subsection{Motivation}

Self-assembly is the ubiquitous process in which smaller objects combine together to form larger complex objects. Recently, it has been demonstrated as an efficient mechanism for bottom-up construction of nanostructures in nanotechnology [5, 11, 13, 14, $24,33,37,38]$. The potential of self-assembly is not limited to nanofabrication. The ability of two-dimensional and three-dimensional assemblies to perform parallel universal computations has been explored in development of self-assembly of DNA tiles as a tool for nanocomputation $[12,19,29,34,36]$. The potential advantage of significantly high circuit density in molecular circuits as compared to the traditional microelectronic circuits, is chief motivation for developments of molecular circuit components $[1,8,17,18,39]$. Molecular scale circuits need the bottom-up approach of self-assembly of DNA tiles to get assembled out of these molecular electronic components. For the selective attachment of the molecular electronic components to particular tiles of DNA tiling, [27] prepared molecular DNA-linked systems, while [4] used directed self-assembly of molecular terrace structures in organic monolayers. Self-assembly has been demonstrated at larger scales (meso-scale) using capillary forces for interactions between meso-scale tiles [3, 21]. However, major hurdle in harnessing the capabilities of algorithmic self-assembly are the errors that occur during the assembly.

\subsection{Prior Work in 2D Tiling Assembly Error-Correction}

In DNA assemblies, incorrect tiles are incorporated in the growing structure with error rates ranging from $1 \%$ to 5\% [32]. There are two approaches to combat the errors. The first is to reduce the inherent error rate by optimizing the physical conditions [30] or using newer molecular mechanisms [7], while the other approach is to improve the tile design so that the total number of errors in the final structure is reduced in spite of the intrinsic error-rate remaining the same [6, 20, 32].

Winfree's pioneering work in error-correction [32] laid the foundations towards improving the tile-design to reduce the errors in assembly. It required replacing a tile by a larger block of tiles. Though it resulted in the total size of assembly to be 4 times for error reduction to $\epsilon^{2}$ and 9 times for error reduction to $\epsilon^{3}$, it paved the way for further work in error-reduction using the concept of redundancy. The basic idea was that an error in the assembly of a tile forced more errors in the neighborhood of that tile, making it extremely prone to detachment, and hence reducing the error. Later, the snaked proof-reading scheme that could correct both growth and nucleation errors in 
the self-assembly was built upon this construction [6]. However, it required replacing a tile by a $k \times k$ block of tiles. Later a method was proposed to control nucleation errors programmably [23]. However, each of these schemes significantly scaled up the overall size of assembly. In applications like molecular fabrication tasks where the scale of final pattern is of critical importance, this scaling up is undesirable. Reif et al. [20] proposed a compact error-resilient tiling schemes in which errors could be reduced to $\epsilon^{2}$ (2-way overlay redundancy) and $\epsilon^{3}$ (3-way overlay redundancy) without increasing the size of the assembly. The analysis of error was done in the equilibrium state of the assembly. Another distinction of this scheme was that it considered the error resilience in the whole pattern and not only in the output row. It means that this scheme had a tendency to remove any incorrectly placed tile from the assembly even if the ongoing computation was not affected by that tile. This is important in the assembly of a nanostructure of desired pattern, where any incorrect placement of any tile is a defect (even though it might not have interfered with the subsequent growth of assembly). But it had its limitations on the Boolean functions that could be used for the error-resilient algorithmic assembly. In particular, it required one of the function to be $X O R$, and for reduction to $\epsilon^{3}$ the additional requirement was that the other function should be input-sensitive to one of the inputs. A Boolean function $f(x)$ is called input-sensitive to a Boolean variable $x$ if whenever $x$ changes $f(x)$ also changes. It is thus a critical challenge to improve these compact error-correction schemes to incorporate any arbitrary Boolean functions. In case that is not possible, it is important to characterize the class of Boolean functions to which these error-correction schemes can be extended. Recently Winfree [25] presented a compact error resilient scheme based on Chen et al. [6]. They also overlooked the errors that did not affect the ongoing computation.

\subsection{The Challenge of 3D Tiling Assembly Error-Correction}

Self-assembly in three dimensions is extremely promising in the field of microelectronics assembly, where independent manipulation of each component is required. It is already being seen as promising candidate for heterogeneous three-dimensional integration of next-generation microsystems [9, 15, 28, 35]. In light of the inherent parallelism, three-dimensional nature and larger range (nanoscale to mesoscale) of application of self-assembly, it has a great potential as tool for building complex systems from microscaled templates. Apart from this, the potential advantages of three-dimensional structures over two-dimensional structures in nanofabrication includes a considerably increased circuit density. Jonoska et al. [10] proposed the use of three-dimensional DNA structures in computing. Simple examples of algorithmic computation in three dimensions includes the generalization of Pascal triangle to 3D [2] and three-dimensional multiplexers (the latter would provide a mechanism for 3D memory addressing with the appropriate affixed molecular electronic components). Analogous to the simulation of a finite state automata through two-dimensional selfassembly, three dimensional self-assembly can be used to simulate a two-dimensional cellular automata, where the third spatial dimension of the 3D tiling is the time step of the cellular automata. The tiles in a horizontal plane will represent the current state of all the cells of a two-dimensional cellular automata, then the tiles assembled in 
horizontal plane on top of it will be states at next time instance. This allows one to derive 3D tiling assemblies from a wide variety of known two-dimensional cellular automata designs, including matrix multiplication, integer multipliers, context free language recognition, etc. Recently crystal structure of three-dimensional DNA lattices formed by self-assembly was demonstrated [16]. The question of fault-tolerance naturally arises with the increasing popularity of self-assembly for construction of three dimensional self-assembled structures. It will be critical to determine how successfully can the error-correction techniques used for two-dimensional assemblies be extended to three-dimensions.

\subsection{The Challenge of Self-Healing Tiling Assemblies}

The one property of biological systems that makes them robust is their ability to self-heal in case of damages. Self-healing is essentially the self-assembly of the constituent elements in the damaged part of a system, so as to repair the damage. It is a very important process in nature. The damage to the living cells can be caused by an external intruder or some mechanical impulse or unfavorable physical conditions. It is an interesting and important challenge to design the DNA tiles that form lattices having the ability to self-heal, thereby imparting them the much desired robustness to withstand environmental damage. Winfree [31] gave a construction in which he replaced a single tile with $3 \times 3$ (for simple assemblies like Sierpinski triangles), $5 \times 5$ (for general assemblies) and $7 \times 7$ (for additional robustness to nucleation errors) block of tiles for self-healing in a two-dimensional assembly. Prior to this work, it was an open problem to find if compact self-healing tile sets could be formed and whether the techniques given by Winfree could be extended to three dimensions.

\subsection{Our Results and Organization of Paper}

In this paper, we follow the notion of compactness as presented in [20], which requires the new error-resilient tiling assembly to be of no larger size than the original assembly. Like [20] we consider any incorrect placement of a tile anywhere in the assembly as an error and aim at reducing them as well, even though these errors might not affect the ongoing computation. As mentioned earlier, this is important for construction of nanostructures of desired pattern. In this paper, the analysis of the error in the assembly is done in the equilibrium state of the assembly. Throughout this paper redundancy based compact error resilient scheme refers to any error resilient scheme that does not scale up the size of the assembly and in which the encodings on the pads of the tiles are used to create redundancy. In the event of an error this redundancy forces more errors, which makes the incorrectly placed tiles and their neighborhoods more unstable and prone to removal from assembly, thereby reducing the error. Also we refer to $k$-expansive error resilient schemes as the error correction schemes that work by replacement of a tile by a block of multiple tiles. In case of three dimensional tiling, we carry forward this notion of redundancy based compact error resilient schemes.

In this paper, we present a comprehensive theory of redundancy based compact error resilient tiling schemes and examine the prospects of constructing compact selfhealing tile sets in two and three-dimensions. The error analysis throughout this paper 


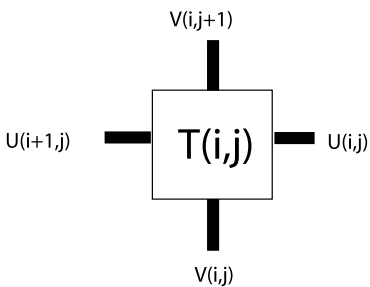

(a)

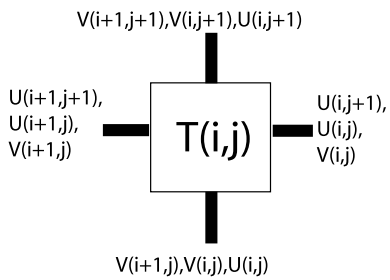

(b)

Fig. 1 (a) Two dimensional algorithmic self-assembly; (b) Construction for error reduction to $\epsilon^{2}$

is in the equilibrium state of the assembly. In Sect. 2, first we present a compact error correction schemes in two dimensional self-assembly that reduces the error from $\epsilon$ to $\epsilon^{2}$ for arbitrary Boolean functions. Then we characterize the class of Boolean functions for which error reduction from $\epsilon$ to $\epsilon^{3}$ is possible using redundancy based compact error resilient schemes. Also we prove that error reduction from $\epsilon$ to $\epsilon^{4}$ is impossible using redundancy based compact error resilient schemes. Next in Sect. 3 we examine three-dimensional self-assembly. First we present a compact error resilient scheme that reduces error to $\epsilon^{2}$ for arbitrary Boolean functions and $\epsilon^{3}$ for a restricted class of input-sensitive Boolean functions. We also prove that error reduction to $\epsilon^{4}$ can not be obtained for arbitrary Boolean functions using redundancy based compact error resilient schemes. In Sect. 4 we extend the idea of Winfree's construction for self-healing in two-dimensions [31] to three-dimensional assembly. In the conclusion, we review our results and state various open problems and conjectures. We conjecture stronger results that error reduction to $\epsilon^{3}$ in three dimensions can not be achieved outside the previously characterized class, and error reduction to $\epsilon^{4}$ is impossible to achieve for any Boolean functions using these error resilient techniques.

\section{Error Correction in Self-Assembly in Two Dimensions}

\subsection{Assembly in Two Dimensions}

We will consider a general assembly problem in two dimensions consisting of the assembly of a two-dimensional Boolean array of size $N \times M$, where the elements of each column are indexed from 0 to $N-1$ from right to left and rows are indexed from 0 to $M-1$ from bottom to top. The bottom row and the rightmost column provide the inputs to the assembly.

For $i=0, \ldots, N-1$ and $j=0, \ldots, M-1$ :

Let $V(i, j)$ be the value of the $i$ th column (from the right) in the $j$ th row (from the bottom). Let $V(i, j+1)$ be the value communicated to the position $(i, j+1)$ and $U(i+1, j)$ be the value communicated to the position $(i+1, j)$. We define $U(i+1, j)=U(i, j) O P_{1} V(i, j)$ and $V(i, j+1)=U(i, j) O P_{2} V(i, j)$ for two Boolean functions $O P_{1}$ and $O P_{2}$.

Figure 1(a) shows a computational tile that can be used for constructing two dimensional self-assembly. Bottom and right pads are the input pads, while the 
pads on top and left are output pads. A pad matches with the neighbor's contiguous pad if the values communicated by these pads are the same. $U(i, j)$ and $V(i, j)$ are the right and bottom input pads, respectively, to the $i$ th column from right and $j$ th row from bottom. Then $U(i+1, j)$ the left output pad is given by $U(i+1, j)=U(i, j) O P_{1} V(i, j)$, while $V(i, j+1)$ the top output pad is given by $V(i, j+1)=U(i, j) O P_{2} V(i, j)$. The collection of tiles required for an assembly is referred to as the tile set for that assembly. Examples of simple two dimensional assemblies: Sierpinski triangle and binary counter, and their respective tile sets, are given in [20]. Highly complex two-dimensional assemblies are possible due to the universal computability of two-dimensional self-assembly [26, 34].

\subsection{The Error Model}

In this paper, we address the mismatch errors between the input and output pads of neighboring tiles in self-assembly. Specifically, a mismatch between the top (or left) output pad of a tile and bottom (or right) input pad of its top (or left) neighbor, is referred as a mismatch error. However, in a kinetic system, an incorrect tile might dissociate from the assembly, due to the lack of inadequate binding support. In case, the incorrect tile does not dissociate but stays in the assembly, then the error stays. Intuitively speaking, in our algorithmic self-assembly model, information flows from right to left and bottom to top. As a result of a mismatch error incorrect information might flow from left to right or bottom to top in the algorithmic assembly. We assume that error probability $\epsilon$ is defined as the probability that there is mismatch between two tiles and they still stay together in the equilibrium. This probability is independent of any other match or mismatch and hence we term this probabilistic model the independent error model. The justification behind the independent error model comes from the definition of $\epsilon$. We have defined $\epsilon$ to be the probability of the event that (i) there is an error of pad binding between a pair of tiles and (ii) these tiles stay attached to each other. The justification is simply that: once these two tiles stay attached to each other, this event (or incorrect pad binding) can not affect other pad binding events between other pairs of tiles. We also want to put emphasis on the correct assembly of all the tiles in the assembly (and hence on the correctness of complete pattern), and not just on the correctness of final output only. There might be wrong placement(s) of tile(s), that do not affect the ongoing computation. But in our error model, we count them as errors and need the error correction schemes to reduce such errors as well. In this way we differ from [25], who overlooked the errors that did not affect the ongoing computation. Consider a tile $T(i, j)$ in a $N \times M$ tiling assembly where $0<i<N-1,0<j<M-1$. We define the neighborhood of a tile $T(i, j)$ as 8 tiles surrounding it, whose coordinates differ from $(i, j)$ by at most 1 . Formally speaking, $\left\{T\left(i^{\prime}, j^{\prime}\right):\left|i^{\prime}-i\right| \leq 1,\left|j^{\prime}-j\right| \leq 1\right\} \backslash\{T(i, j)\}$. Tile $T\left(i^{\prime}, j^{\prime}\right)$ is said to be a-dependent (for assembly dependent) on tile $T(i, j)$ if $i^{\prime} \geq i$ and $j^{\prime} \geq j$ and $a$-independent otherwise. Next we examine the schemes to reduce the errors in self-assembly. To reiterate, throughout this paper, we refer to redundancy based compact error resilient scheme as error reduction scheme, where redundancy is created by encodings in the pads with absolutely no scale up of the assembly. Hence, the computation at position $(i, j)$ is still performed at the same position. However, there is an increase in the number of type of pads for tiles. 
Before we describe, our redundancy based compact error correction methods, it is important to understand their underlying principle. If an error in a tile can force more error(s) in its neighborhood, then the tile and its neighborhood become unstable. As a result, they can dissociate from the assembly with a high probability, before other tiles can assemble around them and lock these erroneous tiles in the assembly. It is important for the further errors to be forced in the neighborhood of erroneous tile so that it does not get locked. If its neighborhood is error-free, then it will get locked and the error will persist in the assembly. Following proposition quantifies this idea.

Proposition 1 Under our independent error model, if an error in a pad in a tile enforces $k$ further mismatches in the assembly in the neighborhood of that tile, then the probability of that error is reduced to $\epsilon^{k+1}$.

Proof If one error guarantees $k$ more errors in its neighborhood, then the probability that the tile and its neighborhood in the assembly will stay together in the equilibrium in spite of these $k+1$ errors is $\epsilon^{k+1}$ (by independent error assumption), which implies the claimed error reduction.

Before we present our results on redundancy based error correction schemes, we would like to emphasize that while referring to self-assembly for Boolean functions $O P_{1}$ and $O P_{2}$, we mean all the tile sets with Boolean functions $O P_{1}$ and $O P_{2}$. Therefore, an impossibility result that there does not exist an error correction scheme for self-assembly for Boolean Functions $O P_{1}$ and $O P_{2}$, means that there does not exist an error correction scheme that works for all the tile sets with Boolean functions $O P_{1}$ and $O P_{2}$.

\subsection{Error Reduction to $\epsilon^{2}$}

It is known that if an error in a tile can guarantee another error in neighborhood, then it reduces the rate of errors from $\epsilon$ to $\epsilon^{2}$ [20, 32]. Next we describe our construction to achieve this goal in the form of Theorem 1.

Theorem 1 There exists a compact error correction scheme that will reduce the error from $\epsilon$ to $\epsilon^{2}$ for two-dimensional algorithmic self-assembly for any arbitrary Boolean functions $O P_{1}$ and $O P_{2}$.

Proof Construction: Before we begin the proof we would like to emphasize the wholeness of the pad. Each side of the tile has one pad in Fig. 1(b), and it encodes the triplet shown in the figure. Disagreement between corresponding elements of two such triplets in any two pads results in the total mismatch between those two pads. Consider the tile with input $U(i, j)$ and $V(i, j)$ at the right and bottom pads respectively, where $0 \leq i<N$ and $0 \leq j<M$. Our goal is to guarantee one more error in the neighborhood of this tile if there is one error. For that, we construct an error checking portion $(V(i, j))$ in the right side pad and one error checking portion $(U(i, j))$ in the bottom pad. We will need corresponding parts in the pads on the top $(U(i, j+1))$ and the left side $(V(i+1, j))$ also, which will match with the error 


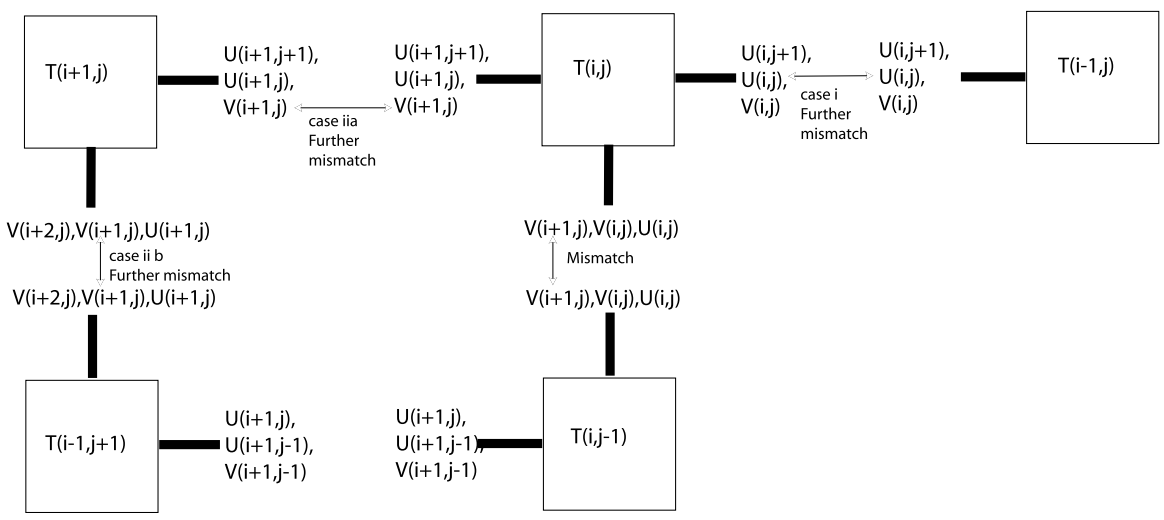

Fig. 2 Case 1(b) A further mismatch is caused by an error in the input pads

checking parts in the bottom pad of the top neighbor $T(i, j+1)$ and right pad of the left neighbor $T(i+1, j)$ respectively. Now since top output pad depends on the value of $U(i, j+1)$ (which is the right input of the top neighbor) we need to incorporate it in our input pads. It is necessary otherwise there will be multiple type of tiles for any given set of input pads. But for successful functioning of algorithmic self-assembly it is required that there should be only one possible tile-type for every set of input pads. So, we need one more portion in the right input pad $(U(i, j+1))$ and hence a corresponding part in the left output pad $(U(i+1, j+1))$. Similarly, the need for another portion in bottom input pad $(V(i+1, j))$ and subsequently, in top output pad $(V(i+1, j+1))$ can be explained.

This completes our description of a tile in our compact error correction scheme. It should be noted that the number of different tile types in this tile set will be 4 times as compared to number of tiles in a tile set without any error-correction. It can be attributed to the two possible values for each of $U(i, j+1)$ and $V(i+1, j)$, for every value of the inputs $U(i, j)$ and $V(i, j)$.

Error-Analysis: We show that if the neighborhood tiles a-independent of $T(i, j)$ are assembled correctly then a pad binding error in any of the input pads in $T(i, j)$ causes an additional mismatch error in its neighborhood in equilibrium. We need to consider only the cases where the pad binding error occurs in either the bottom or the right pad of tile $T(i, j)$. Otherwise, if the error occurs in left (or top) pad of $T(i, j)$ then we can consider the right pad of $T(i+1, j)$ (or bottom) pad of $T(i, j+1)$ for the analysis. The following case analysis provides the required proof.

1. If the bottom pad of $T(i, j)$ has a mismatch:

(a) If $V(i, j)$ on the bottom pad has a mismatch, then $V(i, j)$ on right pad is incorrect, which causes an additional mismatch.

(b) If $V(i, j)$ on the bottom pad is correct and $V(i+1, j)$ on bottom pad has a mismatch, $V(i+1, j)$ on left pad is incorrect (Fig. 2). Now we will prove that it causes a further mismatch by exactly same technique as used by Reif et al. [20]. We have assumed that all the rows and columns that are $a$ independent of tile $T(i, j)$ are correctly assembled so $T(i+1, j-1)$ is correctly assembled and has correct values of its top output pad. Hence $T(i, j)$ 's 
Fig. 3 Case 2(b) of the proof

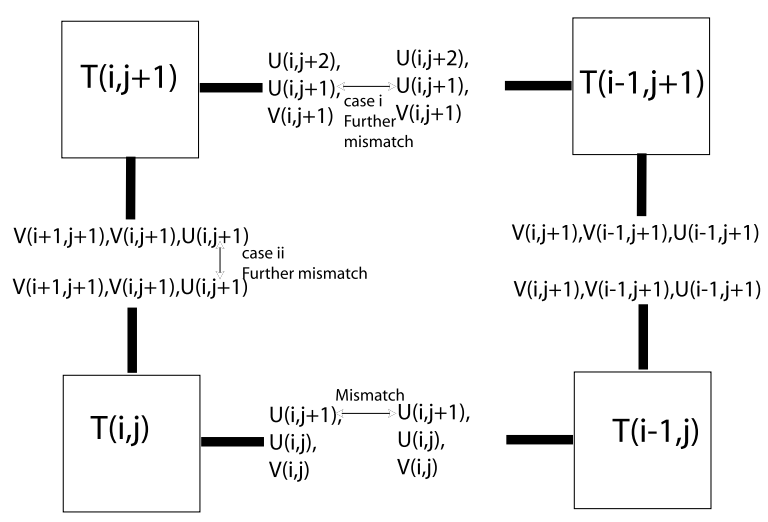

left neighbor $T(i+1, j)$ is dependent upon the incorrect value communicated by the left pad of $T(i, j)$ and correct values communicated by top pad of $T(i+1, j-1)$. Now consider the pads of $T(i+1, j)$. The right pad includes $U(i+1, j+1), U(i+1, j), V(i+1, j)$ and bottom pads include $V(i+2, j), V(i+1, j), U(i+1, j)$. Since the value $V(i+1, j)$ communicated by $T(i+1, j-1)$ is correct and the value $V(i+1, j)$ communicated by $T(i, j)$ is wrong, this implies there will be a mismatch at the right or bottom pad of Tile $T(i+1, j)$.

2. If there is no error in bottom pad, but the right pad of $T(i, j)$ has mismatch:

(a) If $U(i, j)$ on the right pad has a mismatch, then $U(i, j)$ on bottom pad is incorrect, which causes an additional mismatch.

(b) If $U(i, j)$ on right pad is correct but $U(i, j+1)$ on right pad is incorrect, then $U(i, j+1)$ on top output pad is incorrect. Now we will show that it causes a further mismatch as argued above (Fig. 3). Since we assume that all the rows and columns that are $a$-independent of tile $T(i, j)$ are correctly assembled $T(i-1, j+1)$ is correctly assembled and has correct values of its left output pad. Hence $T(i, j)$ 's top neighbor is dependent upon the incorrect value communicated by the top pad of $T(i, j)$ and correct values communicated by left pad of $T(i-1, j+1)$. Now consider the pads of $T(i, j+1)$. The right pad includes $U(i, j+2), U(i, j+1), V(i, j+1)$ and bottom pads include $V(i+1, j+1), V(i, j+1), U(i, j+1)$. Since $V(i+1, j)$ communicated by $T(i-1, j+1)$ is correct and the value $V(i+1, j)$ communicated by $T(i, j)$ is wrong, this implies there will be a mismatch at the right or bottom pad of Tile $T(i, j+1)$.

Hence any mismatch on the right or bottom pad of tile $T(i, j)$ causes one more mismatch in the vicinity of the tile. Together with the Proposition 1 this implies that this scheme can reduce the pad mismatch errors from $\epsilon$ to $\epsilon^{2}$.

\subsection{Error Reduction to $\epsilon^{3}$}

At this point we would like to reiterate that redundancy based compact error resilient scheme refers to any error resilient scheme that does not scale up the assembly and in 
Table 1 An example of the $O P_{1}$ and $O P_{2}$

$\overline{U \quad V \quad U O P_{1} V \quad U O P_{2} V}$

$\begin{array}{llll}0 & 0 & 1 & 0\end{array}$

$\begin{array}{llll}0 & 1 & 1 & 1\end{array}$

$1 \quad 0 \quad 0 \quad 0$

$\begin{array}{llll}1 & 1 & 0 & 1\end{array}$

which only the encodings on the pads of the tiles are used to create redundancy. Also, a Boolean function $f(x)$ is said to be input-sensitive to Boolean input $x$ if it changes for every change in the value of $x$.

Before we proceed with the Error-analysis, it will be useful to understand the function class characterized in the theorems below. Let $O P_{1}$ and $O P_{2}$ be each be two-input Boolean functions such that:

1. $U(i, j) O P_{1} V(i, j)$ is input-sensitive to $U(i, j)$, if $V(i, j)$ is kept constant and $U(i, j) O P_{2} V(i, j)$ is input-sensitive to $V(i, j)$ if $U(i, j)$ is kept constant.

2. When both of them change at least one of the $U(i, j) O P_{1} V(i, j)$ or $U(i, j) O P_{2} V(i, j)$ should also change.

For $U(i, j)=0$, there are 2 possible assignments to $U(i, j) O P_{1} V(i, j)$ maintaining its input-sensitivity to $V(i, j)$. Similarly, for $U=1$ there are 2 possible assignments to $U(i, j) O P_{1} V(i, j)$ conditioned to its input-sensitivity to $V(i, j)$. Similarly for $V(i, j)=0$ and $V(i, j)=1$ there are 2 independent assignments each. But among these half of the assignments do not satisfy the second condition. Hence the total number of Boolean functions in this class are 8. An example of such a function is given in the Table 1.

Define the pair of Boolean functions $O P_{1}$ and $O P_{2}$ to be pairwise input-sensitive if at least one of the $U(i+1, j)$ or $V(i, j+1)$ changes for any change in $U(i, j)$ or $V(i, j)$.

Theorem 2 For arbitrary Boolean functions $O P_{1}$ and $O P_{2}$, there does not exist any redundancy based compact error resilient scheme for two-dimensional self-assembly that can reduce the error from $\epsilon$ to $\epsilon^{3}$.

Proof For errors to reduce from $\epsilon$ to $\epsilon^{3}$, an error in any input pad, say $V(i, j)$ should cause two further mismatches in the neighborhood. At least one of those mismatches should be caused because of an error on one of the output pads. It should be noted that if $O P_{1}$ and $O P_{2}$ are arbitrary Boolean functions then the output $U(i+1, j)$ or $V(i, j+1)$ cannot be guaranteed to be wrong for incorrect value of $V(i, j)$. Hence, in at least one of the output pads an additional error checking portion $f(V(i, j))$ (that is input-sensitive to $V(i, j)$ and hence can reflect the error in $V(i, j))$ is required. It can be located on the top or left output pad.

- Assume that $f(V(i, j))$ is located on top pad, which implies $f(V(i, j-1))$ is located on the bottom pad (shown by arrows in Fig. 4(a)).

1. If $V(i, j-1)$ does not exist within the input pads, then we need to consider the case when $f(V(i, j-1))$ has a mismatch. Since we require two further errors 
Fig. 4 Schematic to illustrate the proof of Theorem 2

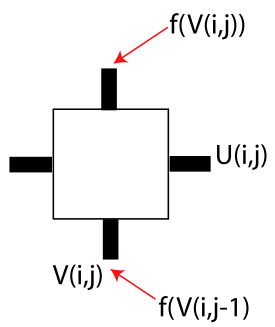

(a)

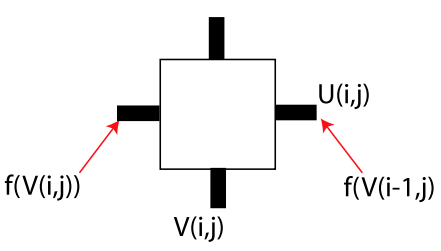

(b)

in the neighborhood of $T(i, j)$, as argued above it requires an additional error checking function $g(f(V(i, j-1)))$ (that is input-sensitive to $f(V(i, j-1))$ ) on at least one of the top or left output pad.

2. If $V(i, j-1)$ exists in the input pads, then in case when $V(i, j-1)$ is mismatched, and two further errors in the neighborhood of $T(i, j)$ are required, it needs an additional error checking function $g^{\prime}(V(i, j-1))$ (that is inputsensitive to $V(i, j-1))$ on at least one of the top or left output pad.

- Assume that $f(V(i, j))$ is located on left pad, which implies $f(V(i-1, j))$ is located on the right pad (shown by arrows in Fig. 4(b)).

1. If $V(i-1, j)$ does not exist within the input pads, we need to consider the case when $f(V(i-1, j))$ is mismatched. Since two further errors are required, as argued above it requires an additional error checking function $h(f(V(i-1, j)))$ (that is input-sensitive to $f(V(i-1, j)))$ to be located on at least one of the top or left output pad.

2. If $V(i-1, j)$ exists in the input pads, then in case when $V(i-1, j)$ is mismatched, and two further errors are required, it requires an additional error checking function $h^{\prime}(V(i-1, j))$ (that is input-sensitive to $V(i-1, j)$ ) to be present on at least one of the top or left output pads.

Hence, an additional error checking pad $\left(g(f(V(i, j-1))), g^{\prime}(V(i, j-1))\right.$ or $h(f(V(i-1, j)))$ or $h(V(i-1, j)))$ is required on at least one of the output pads. Arguing in the same manner as above we conclude that this cycle will keep on repeating. Hence, it is not possible to construct a tile with a bounded number of parameters in the pads such that a mismatch results in two more mismatches in the neighborhood of the tile in a two-dimensional assembly. Combining it with Proposition 1 we conclude that redundancy based compact error resilient schemes can not reduce error from $\epsilon$ to $\epsilon^{3}$.

However, it will be proved that for a rather restricted class of Boolean functions $O P_{1}$ and $O P_{2}$, error can be reduced to $\epsilon^{3}$ by using the construction of Fig. 1(b), which is stated as Theorem 3 .

Theorem 3 For Boolean functions $O P_{1}$ and $O P_{2}$ which are pairwise input-sensitive, there exists a redundancy based compact error resilience scheme that can reduce the error to $\epsilon^{3}$. 
Proof For our proof, we will use the scheme shown in Fig. 1(b). If $O P_{1}$ and $O P_{2}$ are restricted to be as described, and if the neighborhood tiles that are $a$-independent of $T(i, j)$ are assembled correctly, then a pad binding error in any of the input pads in $T(i, j)$ causes two additional mismatch errors in its neighborhood. As explained earlier, we need to consider only the cases where the pad binding error occurs in either the bottom or the right pad of tile $T(i, j)$. The following case analysis provides the required proof.

1. If the bottom pad of $T(i, j)$ has a mismatch:

(a) If $V(i, j)$ in bottom pad of $T(i, j)$ has a mismatch, then the $V(i, j)$ in the right pad of $T(i, j)$ is incorrect. This causes a mismatch because according to our assumption, all the tiles $a$-independent of $T(i, j)$ are assembled correctly. Also:

i. If $U(i, j)$ on right pad is correct, $V(i, j+1)$ on top pad is incorrectly computed because of restrictions on $O P_{1}$ and $O P_{2}$. This will cause further mismatch at the right or bottom pad of the top neighbor $T(i, j+1)$, as argued in the proof of Theorem 1 .

ii. If $U(i, j)$ on right pad has a pad-mismatch, then at least one of the $V(i, j+1)$ on top pad or $U(i+1, j)$ on left pad is incorrectly computed, because of the restrictions on $O P_{1}$ and $O P_{2}$. This will cause a further mismatch at right or bottom pad of the left neighbor $(T(i+1, j))$ or top neighbor $(T(i, j+1))$ in the same way as argued earlier.

(b) If $V(i, j)$ on bottom pad is correct and $V(i+1, j)$ on bottom pad has mismatch, then $V(i+1, j)$ on the left pad is incorrect, which causes a further mismatch in the right or bottom pad of the left neighbor $T(i+1, j)$. Also:

i. If $U(i, j)$ on right pad is incorrect, then this causes a mismatch on the right pad of $T(i, j)$, because according to our assumption, all the tiles $a$-independent of $T(i, j)$ are assembled correctly.

ii. If $U(i, j)$ on right pad is correct, then $U(i+1, j)$ on left output pad is correct. But since $V(i+1, j)$ has a mismatch, $V(i+1, j+1)$ on the top pad is incorrectly computed, because of the restriction on $O P_{1}$ and $O P_{2}$. This causes a further mismatch on the bottom or the right pad of the top neighbor tile $T(i, j+1)$.

2. If there is no error in the bottom pad and there is mismatch in right pad:

(a) If $U(i, j)$ on the right pad has a pad-mismatch, then at bottom $U(i, j)$ is incorrect, and causes a mismatch. However since $V(i, j)$ is correct on the bottom pad so $U(i+1, j)$ on the left pad is incorrectly computed because of the restriction on $O P_{1}$ and $O P_{2}$. This causes a further mismatch on right or bottom pad of left neighbor as explained earlier.

(b) If $U(i, j)$ on right pad is correct and $U(i, j+1)$ has a mismatch, then $U(i, j+1)$ on top pad is incorrect, which causes a further mismatch in right or bottom pad of the top neighbor tile $T(i, j+1)$. Also since $V(i, j)$ is correct, $V(i, j+1)$ is also correct, and hence $U(i+1, j+1)$ on left pad is incorrectly computed because of restriction on $O P_{1}$ and $O P_{2}$. This causes a further mismatch in the right or bottom pad of the left neighboring tile $T(i+1, j)$. 
Hence any mismatch on the right or bottom side of the tile $T(i, j)$ causes two further mismatches in the vicinity of tile $T(i, j)$. This results in error reduction from $\epsilon$ to $\epsilon^{3}$ using Proposition 1 .

We conjecture the following stronger result, which is currently an open question to be proved or disproved.

Conjecture 1 For any combination of Boolean functions $O P_{1}$ and $O P_{2}$ outside the restricted class of Theorem 3, there exists no redundancy based compact error correction schemes that can reduce the error from $\epsilon$ to $\epsilon^{3}$ in two-dimensional self-assembly.

\subsection{Error Reduction to $\epsilon^{4}$}

Theorem 4 For any Boolean functions $O P_{1}$ and $O P_{2}$, there exists no redundancy based compact error correction scheme that can reduce error from $\epsilon$ to $\epsilon^{4}$ in twodimensional self-assembly.

Proof For the reduction of error from $\epsilon$ to $\epsilon^{4}$, a mismatch in any input pad should cause 3 more mismatches. It means that for any error in one of the input pads both the output pads should have errors. In case an output pad requires any additional error checking portion to detect an error in an input, then by arguments similar to the proof of Theorem 2, it can be shown that such a tile cannot be constructed.

Hence, the only possibility is when, the left and top outputs $U(i+1, j)$ and $V(i, j+1)$ both change for any change in the input $U(i, j)$ or $V(i, j)$. This means that we have different values for each of $U(i+1, j)$ and $V(i, j+1)$ for 4 different values of input pair, which is not possible as $U(i+1, j)$ and $V(i, j+1)$ are Booleans.

\section{Error Correction in Self-Assemblies in Three Dimensions}

Three dimensional self-assembly is being described as the most promising tool for heterogeneous integration of next generation microsystems. Its potential to build complex systems from microscale templates can not be overlooked $[9,15,28,35]$. Besides the assembled three-dimensional structures can be extremely useful in computations [10]. It is possible to simulate a two-dimensional cellular automata, using three-dimensional self-assembly, which then paves way to perform a rich class of computations including matrix multiplication, integer multiplications, context-free language recognition etc.

\subsection{Assembly in Three Dimensions}

The assembly problem in three-dimensions can be generalized from the twodimensional assembly as the assembly of a three-dimensional Boolean array of size $N \times M \times P$, where the elements are indexed from 0 to $N-1$ from right to left, 0 to $M-1$ from bottom to top, and 0 to $P-1$ from front to back. The bottommost 
Fig. 5 Three dimensional algorithmic self-assembly

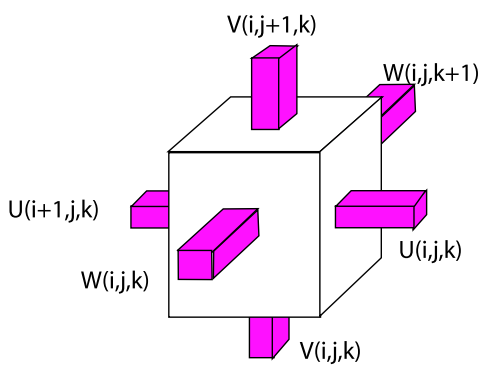

horizontal plane, and rightmost and frontmost vertical planes provide the inputs to the assembly.

Let $V(i, j, k)$ be the value of element at $i$-th position from right, $j$-th position from bottom, and $k$-th position from front. Let $U(i+1, j, k)$ be the value communicated to the position $(i+1, j, k), V(i, j+1, k)$ be communicated to the position $(i, j+1, k)$, and $W(i, j, k+1)$ be communicated to the position $(i, j, k+1)$. We define $U(i+1, j, k)=f_{1}(U(i, j, k), V(i, j, k), W(i, j, k)), V(i, j+1, k)=$ $f_{2}(U(i, j, k), V(i, j, k), W(i, j, k)), \quad W(i, j, k+1)=f_{3}(U(i, j, k), \quad V(i, j, k)$, $W(i, j, k))$ for Boolean functions $f_{1}, f_{2}$, and $f_{3}$.

Figure 5 shows a computational tile that can be used for construction of threedimensional assembly. Right, bottom and front pads are the input pads, while the pads on left, top and back are output pads. As in two-dimensional assembly, a pad matches with the neighbor's contiguous pad if the values communicated by these pads are the same. $U(i, j, k), V(i, j, k)$ and $W(i, j, k)$ are the right, bottom and front input pads, respectively, to the tile located at position $(i, j, k)$. Then $U(i+1, j, k), V(i, j+1, k)$ and $W(i, j, k+1)$ are the left, top and back output pads, respectively, of the tile $T(i, j, k)$. Also, $U(i+1, j, k)=f_{1}(U(i, j, k), V(i, j, k), W(i, j, k)), V(i, j+1, k)=$ $f_{2}(U(i, j, k), \quad V(i, j, k), W(i, j, k)), W(i, j, k+1)=f_{3}(U(i, j, k), \quad V(i, j, k)$, $W(i, j, k)$ ) where $f_{1}, f_{2}$ and $f_{3}$ are the ternary Boolean functions that take as input three Boolean values and give a Boolean output. It is assumed that initially a frame is assembled, with $M \times P$ tiles in rightmost plane, $N \times P$ tiles in bottommost plane and $N \times P$ tiles in frontmost plane. Next we examine the error resilience in three-dimensional self-assembly.

\subsection{The Error Model}

We extend the error model in two-dimensions to three-dimensional assembly in an obvious way. We follow the independent error model for three dimensional assembly. We also want to emphasize on the correct assembly of all the tiles in the assembly (and hence on the correctness of complete pattern), and not just on the correctness of final output only. We want to emphasize that the error analysis is done in the equilibrium state of the assembly. Consider a tile $T(i, j, k)$ in a $N \times M \times P$ tiling assembly where $0<i<N-1,0<j<M-1$, and $0<k<P-1$. We define the neighborhood of a tile $T(i, j, k)$ as 26 tiles surrounding it, whose coordinates differ from $(i, j, k)$ by at most 1 . Formally speaking, $\left\{T\left(i^{\prime}, j^{\prime}, k^{\prime}\right):\left|i^{\prime}-i\right| \leq 1\right.$, $\left.\left|j^{\prime}-j\right| \leq 1,\left|k^{\prime}-k\right| \leq 1\right\} \backslash\{T(i, j, k)\}$. Tile $T\left(i^{\prime}, j^{\prime}, k^{\prime}\right)$ is said to be a-dependent 


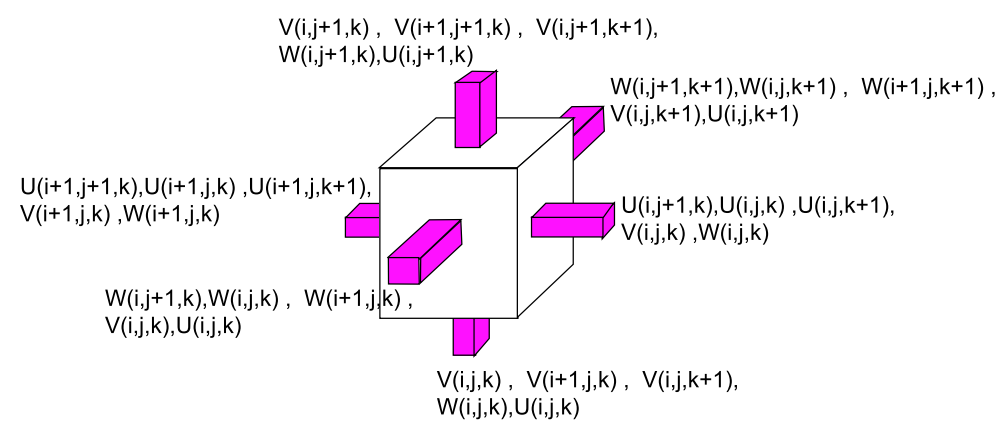

Fig. 6 Construction for error reduction to $\epsilon^{2}$

on tile $T(i, j, k)$ if $i^{\prime} \geq i, j^{\prime} \geq j$, and $k^{\prime} \geq k$ and $a$-independent otherwise. Next we examine the schemes to reduce the errors in self-assembly. As mentioned earlier redundancy based compact error resilient scheme refers to an error resilient scheme that does not scale up the assembly and in which the encodings on the pads of the tiles are used to create redundancy.

\subsection{Error Reduction to $\epsilon^{2}$}

Theorem 5 There exists a redundancy based compact error resilient tiling scheme in three dimensional assembly which can reduce the error from $\epsilon$ to $\epsilon^{2}$ for any arbitrary Boolean functions $f_{1}, f_{2}$, and $f_{3}$, and it is shown in Fig. 6.

Proof Construction: Before we describe the construction, we would like to emphasize on the wholeness of pad. Each side of the tile has one pad in Fig. 6, that encodes a 5-tuple as shown in the figure. Disagreement between corresponding elements of two such 5-tuples in any two pads results in the total mismatch between those two pads. Consider the tile $T(i, j, k)$ with inputs $U(i, j, k), V(i, j, k)$ and $W(i, j, k)$ on the right, bottom and front pads respectively. Our goal is to guarantee one more error in the vicinity of this tile if there is one error in any of the input pads.

We add error checking portions to the right, bottom and front pads as shown in Fig. 6: $V(i, j, k)$ and $W(i, j, k)$ on right pad, $W(i, j, k)$ and $U(i, j, k)$ on bottom pad and $U(i, j, k)$ and $V(i, j, k)$ on front pad. Corresponding to these, we need to add $V(i+1, j, k)$ and $W(i+1, j, k)$ on left pad, $W(i, j+1, k)$ and $U(i, j+1, k)$ on top pad and $U(i, j, k+1)$ and $V(i, j, k+1)$ on back pad, as explained in the case of two-dimensional tile.

As described in two-dimensional assembly, every value in the output pads should be uniquely derivable from the values on the input pads. For $V(i+1, j, k)$ and $W(i+1, j, k)$ on the left pad we add $V(i+1, j, k)$ on the bottom pad, and $W(i+1, j, k)$ on the front pad. For $U(i, j+1, k)$ and $W(i, j+1, k)$ on the top pad, we add $U(i, j+1, k)$ to the right pad and $W(i, j+1, k)$ to the front pad. For $U(i, j, k+1)$ and $V(i, j, k+1)$ on the back pad, we add $U(i, j, k+1)$ to the right pad and $V(i, j, k+1)$ to the bottom pad. The construction is complete with addition of $U(i+1, j+1, k)$ and $U(i+1, j, k+1)$ to left pad, $V(i+1, j+1, k)$ and 
$V(i, j+1, k+1)$ to top pad, and $W(i+1, j, k+1)$ and $W(i, j+1, k+1)$ to back pad. It can be verified that all the values on output pads are uniquely derivable from the values on the input pads.

This completes our description of a tile in our compact error correction scheme. It should be noted that the number of different tile types in this tile set will be 64 times as compared to number of tiles in a tile set without any error-correction. It can be attributed to the two values for each of the $U(i, j+1, k), U(i, j, k+1)$, $V(i+1, j, k), V(i, j, k+1), W(i+1, j, k)$ and $W(i, j+1, k)$, for every value of the inputs $U(i, j, k), V(i, j, k)$ and $W(i, j, k)$.

Error-Analysis: We show that if the neighborhood tiles a-independent of $T(i, j, k)$ are assembled correctly then a pad binding error in any of the input pads in $T(i, j, k)$ causes at least one additional mismatch error in its neighborhood in equilibrium. We need to consider only the cases where the pad binding error occurs in the bottom, the right or the front pad of tile $T(i, j, k)$. Otherwise, if the error occurs in top, left or back pad of $T(i, j, k)$ then we can consider the right pad of $T(i+1, j, k)$, bottom pad of $T(i, j+1, k)$ or front pad of $T(i, j, k+1)$, respectively, for the analysis. The following case analysis provides the required proof.

1. If the bottom pad of $T(i, j, k)$ has a mismatch:

(a) If $V(i, j, k)$ on the bottom pad has a mismatch, then the values of $V(i, j, k)$ on the right and front pads are incorrect, which causes mismatches in right and front pads of $T(i, j, k)$.

(b) If $V(i, j, k)$ on the bottom pad is correct and $V(i+1, j, k)$ on bottom pad of $T(i, j, k)$ has a mismatch, then $V(i+1, j, k)$ on left pad is incorrect. Now we will prove that it causes a further mismatch by exactly same technique as used by Reif et al. [20]. We have assumed that all the tiles that are $a$-independent of tile $T(i, j, k)$ are correctly assembled so $T(i+1, j-1, k)$ is correctly assembled and has correct values of its top output pad. Hence $T(i, j, k)$ 's left neighbor $T(i+1, j, k)$ is dependent upon the incorrect value communicated by the left pad of $T(i, j, k)$ and correct values communicated by top pad of $T(i+1, j-1, k)$. Now consider the pads of $T(i+1, j, k)$. The right pad includes $U(i+1, j+1, k), U(i+1, j, k), U(i+1, j, k+1), V(i+1, j, k)$, $W(i+1, j, k)$ and bottom pads include $V(i+1, j, k), V(i+2, j, k)$, $V(i+1, j, k+1), W(i+1, j, k), U(i+1, j, k)$. Since the value $V(i+1, j, k)$ communicated by $T(i+1, j-1, k)$ is correct and the value $V(i+1, j, k)$ communicated by $T(i, j, k)$ is wrong, this implies there will be a mismatch at the right or bottom pad of Tile $T(i+1, j, k)$.

(c) If $V(i, j, k)$ and $V(i+1, j, k)$ on the bottom pad are correct and $V(i, j, k+1)$ on the bottom pad has a mismatch, then the value of $V(i, j, k+1)$ on the back pad is incorrect. Now we will show that it causes a further mismatch as argued above. Since we assume that all the tiles that are $a$-independent of tile $T(i, j, k)$ are correctly assembled so $T(i, j-1, k+1)$ is correctly assembled and has correct values of its top output pad. Hence $T(i, j, k)$ 's back neighbor $T(i, j, k+1)$ is dependent upon the incorrect value communicated by the back pad of $T(i, j, k)$ and correct values communicated by top pad of $T(i, j-1, k+1)$. Now consider the pads of $T(i, j, k+1)$. The front pad includes $W(i, j+1, k+1), W(i, j, k+1), W(i+1, j, k+1), V(i, j, k+1)$, 
$U(i, j, k+1)$ and bottom pads include $V(i, j, k+1), V(i+1, j, k+1)$, $V(i, j, k+2), W(i, j, k+1), U(i, j, k+1)$. Since the value $V(i, j, k+1)$ communicated by $T(i, j-1, k+1)$ is correct and the value $V(i, j, k+1)$ communicated by $T(i, j, k)$ is wrong, this implies there will be a mismatch at the front or bottom pad of Tile $T(i, j, k+1)$.

2. If there is no error in bottom pad, but the right pad of $T(i, j, k)$ has mismatch:

(a) If $U(i, j, k)$ on the right pad has a mismatch, then the values of $U(i, j, k)$ on bottom pad and front pad are incorrect, which causes two additional mismatches.

(b) If $U(i, j, k)$ on right pad is correct but $U(i, j+1, k)$ on right pad is incorrect, then $U(i, j+1, k)$ on top output pad is incorrect. By the assumption of errorfree assembly of a-independent tiles, we can argue as before that the value $U(i, j+1, k)$ communicated by left pad of $T(i-1, j+1, k)$ is correct and the value $U(i, j+1, k)$ communicated by top pad of $T(i, j, k)$ is wrong, implying that there will be a mismatch at the right or bottom pad of Tile $T(i, j+1, k)$.

(c) If $U(i, j, k)$ and $U(i, j+1, k)$ on right pad are correct but $U(i, j, k+1)$ on right pad is incorrect, then $U(i, j, k+1)$ on back output pad is incorrect. By the assumption of error-free assembly of a-independent tiles, we can argue as before that the value $U(i, j, k+1)$ communicated by left pad of $T(i-1, j, k+1)$ is correct and the value $U(i, j, k+1)$ communicated by back pad of $T(i, j, k)$ is wrong, implying that there will be a mismatch at the right or front pad of Tile $T(i, j, k+1)$.

3. If there is no error in bottom or right pad of $T(i, j, k)$, but the front pad of $T(i, j, k)$ has mismatch:

(a) If $W(i, j, k)$ on the front pad has a mismatch, then the values of $W(i, j, k)$ on bottom pad and right pad are incorrect, which causes two additional mismatches.

(b) If $W(i, j, k)$ on front pad is correct but $W(i, j+1, k)$ on front pad is incorrect, then $W(i, j+1, k)$ on top output pad is incorrect. By the assumption of errorfree assembly of a-independent tiles, we can argue as before that the value $W(i, j+1, k)$ communicated by back pad of $T(i, j+1, k-1)$ is correct and the value $W(i, j+1, k)$ communicated by top pad of $T(i, j, k)$ is wrong, implying that there will be a mismatch at the front or bottom pad of Tile $T(i, j+1, k)$.

(c) If $W(i, j, k)$ and $W(i, j+1, k)$ on front pad are correct but $W(i+1, j, k)$ on front pad is incorrect, then $W(i+1, j, k)$ on left output pad is incorrect. By the assumption of error-free assembly of a-independent tiles, we can argue as before that the value $W(i+1, j, k)$ communicated by back pad of $T(i+1, j, k-1)$ is correct and the value $W(i+1, j, k)$ communicated by left pad of $T(i, j, k)$ is wrong, implying that there will be a mismatch at the right or front pad of Tile $T(i+1, j, k)$.

Hence any mismatch on the right, bottom or front pad of tile $T(i, j, k)$ causes at least one more mismatch in the vicinity of the tile. Together with the Proposition 1 this implies that this scheme can reduce the pad mismatch errors from $\epsilon$ to $\epsilon^{2}$. 


\subsection{Error Reduction to $\epsilon^{3}$}

Theorem 6 If Boolean functions $f_{1}, f_{2}$, and $f_{3}$ satisfy the following conditions:

- For fixed $V(i, j, k)$ and $W(i, j, k), f_{1}(U, V, W)$ is input-sensitive to $U(i, j, k)$.

- For fixed $U(i, j, k)$ and $W(i, j, k), f_{2}(U, V, W)$ is input-sensitive to $V(i, j, k)$.

- For fixed $U(i, j, k)$ and $V(i, j, k), f_{3}(U, V, W)$ is input-sensitive to $W(i, j, k)$.

Then there exists a compact error resilient scheme to reduce error from $\epsilon$ to $\epsilon^{3}$ for three-dimensional self-assembly, and it is shown in Fig. 6.

Proof If $f_{1}, f_{2}$, and $f_{3}$ are as given in the theorem, and if the neighborhood tiles that are a-independent of $T(i, j, k)$ are assembled correctly, then a pad binding error in any of the input pads in $T(i, j, k)$ causes at least two additional mismatch errors in its neighborhood. As explained earlier, we need to consider only the cases where the pad binding error occurs in either the bottom, front or the right pad of tile $T(i, j)$. The following case analysis provides the required proof.

1. If the bottom pad of $T(i, j, k)$ has a mismatch:

(a) If $V(i, j, k)$ on the bottom pad has a mismatch, then the values of $V(i, j, k)$ is incorrect in right and front pads, which causes two further mismatches in the right and front pads of $T(i, j, k)$.

(b) If $V(i, j, k)$ on the bottom pad is correct but $V(i+1, j, k)$ on the bottom pad of $T(i, j, k)$ has a mismatch, then the value of $V(i+1, j, k)$ on left pad is incorrect. This causes a mismatch in the right or bottom pad of the left neighboring tile $T(i+1, j, k)$, as argued earlier using the assumption of errorfree assembly of a-independent tiles in the neighborhood of $T(i, j, k)$. Now there are two cases:

i. $U(i, j, k)$ in the right pad, $W(i, j, k)$ in the front pad, or $W(i+1, j, k)$ in the front pad of tile $T(i, j, k)$ has a mismatch. Thus, there is a further mismatches in the neighborhood of tile $T(i, j, k)$.

ii. All three of the $U(i, j, k)$ in the right pad, and $W(i, j, k)$ and $W(i+$ $1, j, k)$ in the front pad of tile $T(i, j, k)$ are correct. Since $V(i, j, k)$, $U(i, j, k)$, and $W(i, j, k)$ are correct in tile $T(i, j, k), U(i+1, j, k)$ on the left pad is computed correctly. By the condition on $f_{1}, f_{2}$ and $f_{3}$, correct value of $U(i+1, j, k)$ and $W(i+1, j, k)$ and incorrect value of $V(i+1, j, k)$ results in incorrect computation of $V(i+1, j+1, k)$ on the top pad. By the assumption that tiles $a$-independent of $T(i, j, k)$ are assembled correctly, and as argued earlier, it can be proved that there will be another mismatch in the neighborhood of $T(i, j, k)$.

(c) If $V(i, j, k)$ and $V(i+1, j, k)$ on the bottom pad are correct, but $V(i, j, k+1)$ on the bottom pad of $T(i, j, k)$ has a mismatch, then the value of $V(i, j, k+1)$ on the back pad is incorrect. This causes a mismatch in the front or bottom pad of the back neighboring tile $T(i, j, k+1)$. Now there are two cases:

i. $U(i, j, k)$ in the right pad, $U(i, j, k+1)$ in the right pad or $W(i, j, k)$ in front pad has a mismatch. Thus, there is a further mismatch in the neighborhood of $T(i, j, k)$. 
ii. All three of the $U(i, j, k)$ in the right pad, $U(i, j, k+1)$ in the right pad and $W(i, j, k)$ in front pad in tile $T(i, j, k)$ are correct. This results in the correct computation of $W(i, j, k+1)$. By the conditions on $f_{1}, f_{2}$ and $f_{3}$, correct value of $W(i, j, k+1)$ and $U(i, j, k+1)$ and incorrect $V(i, j, k+1)$ results in the incorrect computation of $V(i, j+1, k+1)$ on the top pad. By the assumption that tiles $a$-independent of $T(i, j, k)$ are assembled correctly, and as argued earlier, it can be proved that there will be another mismatch in the neighborhood of $T(i, j, k)$.

2. If there is no mismatch in the bottom pad of tile $T(i, j, k)$ but its right pad has a mismatch:

(a) If $U(i, j, k)$ on the right pad has a mismatch, then the values of $U(i, j, k)$ is incorrect in bottom and front pads, which causes two further mismatches in the right and front pads of $T(i, j, k)$.

(b) If $U(i, j, k)$ on the right pad is correct, but $U(i, j+1, k)$ on the right pad of $T(i, j, k)$ has a mismatch, then the value of $U(i, j+1, k)$ on the top pad is incorrect. This causes a mismatch in the right or bottom pad of the top neighboring tile $T(i+1, j, k)$, due to the assumption of error-free assembly of $a$-independent tiles in the neighborhood of $T(i, j, k)$. Now there are two cases:

i. $W(i, j, k)$ or $W(i, j+1, k)$ in the front pad of tile $T(i, j, k)$ has a mismatch. Thus, there is a further mismatches in the neighborhood of tile $T(i, j, k)$.

ii. Both $W(i, j, k)$ and $W(i, j+1, k)$ in the front pad of tile $T(i, j, k)$ are correct. Since $V(i, j, k), U(i, j, k)$, and $W(i, j, k)$ are correct in tile $T(i, j, k), V(i, j+1, k)$ is computed correctly. By the condition on $f_{1}, f_{2}$ and $f_{3}$, correct value of $V(i, j+1, k)$ and $W(i, j+1, k)$ and incorrect value of $U(i, j+1, k)$ results in incorrect computation of $U(i+1, j+1, k)$ on the left pad. By the assumption that tiles $a$ independent of $T(i, j, k)$ are assembled correctly, and as argued earlier, it can be proved that there will be another mismatch in the neighborhood of $T(i, j, k)$.

(c) If $U(i, j, k)$ and $U(i, j+1, k)$ on the right pad are correct, but $U(i, j, k+1)$ on the right pad of $T(i, j, k)$ has a mismatch, then the value of $U(i, j, k+1)$ on the back pad is incorrect. This causes a mismatch in the front or bottom pad of the back neighboring tile $T(i, j, k+1)$. Now there are two cases:

i. $W(i, j, k)$ in front pad has a mismatch. Thus, there is a further mismatch in the neighborhood of $T(i, j, k)$.

ii. $W(i, j, k)$ in front pad in tile $T(i, j, k)$ is correct. This results in the correct computation of $W(i, j, k+1)$. By the conditions on $f_{1}, f_{2}$ and $f_{3}$, correct value of $W(i, j, k+1)$ and $V(i, j, k+1)$ and incorrect $U(i, j, k+1)$ results in the incorrect computation of $V(i, j+1, k+1)$ on the top pad. By the assumption that tiles $a$-independent of $T(i, j, k)$ are assembled correctly, and as argued earlier, it can be proved that there will be another mismatch in the neighborhood of $T(i, j, k)$.

3. If there is no error in the bottom pad or right pad of tile $T(i, j, k)$, but the front pad has a mismatch: 
(a) If $W(i, j, k)$ on the front pad has a mismatch, then the values of $W(i, j, k)$ is incorrect in bottom and right pads, which causes two further mismatches in the bottom and right pads of $T(i, j, k)$.

(b) If $W(i, j, k)$ on the front pad is correct, but $W(i, j+1, k)$ on the front pad of $T(i, j, k)$ has a mismatch, then the value of $W(i, j+1, k)$ on the top pad is incorrect. This causes a mismatch in the front or bottom pad of the top neighboring tile $T(i+1, j, k)$, due to the assumption of errorfree assembly of $a$-independent tiles in the neighborhood of $T(i, j, k)$. Since $V(i, j, k), U(i, j, k)$, and $W(i, j, k)$ are correct in tile $T(i, j, k), V(i, j+1, k)$ is computed correctly. By the condition on $f_{1}, f_{2}$ and $f_{3}$, correct value of $V(i, j+1, k)$ and $U(i, j+1, k)$ and incorrect value of $W(i, j+1, k)$ results in incorrect computation of $U(i+1, j+1, k)$ on the left pad. By the assumption that tiles $a$-independent of $T(i, j, k)$ are assembled correctly, and as argued earlier, it can be proved that there will be another mismatch in the neighborhood of $T(i, j, k)$.

(c) If $W(i, j, k)$ and $W(i, j+1, k)$ on the front pad are correct, but $W(i+1, j, k)$ on the front pad of $T(i, j, k)$ has a mismatch, then the value of $W(i+1, j, k)$ on the left pad is incorrect. This causes a mismatch in the right or bottom pad of the left neighboring tile $T(i+1, j, k)$. Correct values of $U(i, j, k)$, $V(i, j, k)$, and $W(i, j, k)$ results in the correct computation of $U(i+1, j, k)$. By the conditions on $f_{1}, f_{2}$ and $f_{3}$, correct value of $U(i+1, j, k)$ and $V(i+1, j, k)$ and incorrect $W(i+1, j, k)$ results in the incorrect computation of $V(i, j+1, k+1)$ on the top pad. By the assumption that tiles $a$-independent of $T(i, j, k)$ are assembled correctly, and as argued earlier, it can be proved that there will be another mismatch in the neighborhood of $T(i, j, k)$.

Hence any mismatch on the bottom, right or front side of the tile $T(i, j, k)$ causes two further mismatches in the vicinity of tile $T(i, j, k)$ and this results in error reduction from $\epsilon$ to $\epsilon^{3}$.

\subsection{Error Reduction to $\epsilon^{4}$}

Theorem 7 For arbitrary Boolean functions $f_{1}, f_{2}$, and $f_{3}$, there exists no redundancy based compact error resilient scheme that can reduce error from $\epsilon$ to $\epsilon^{4}$ in three-dimensional self-assembly.

Proof For errors to reduce from $\epsilon$ to $\epsilon^{4}$, an error in any input pad, say $V(i, j, k)$ should cause three further mismatches in the neighborhood. At least one of those mismatches should be caused because of an error on one of the output pads. It should be noted that if the Boolean functions $f_{1}, f_{2}$ and $f_{3}$ are arbitrary Boolean functions then the outputs $U(i+1, j, k), V(i, j+1, k)$ or $W(i, j, k+1)$ cannot be guaranteed to be wrong for incorrect value of $V(i, j, k)$. Hence, in at least one of the output pads an additional error checking portion $f(V(i, j, k)$ ) (that is input-sensitive to $V(i, j, k)$ and hence can reflect the error in $V(i, j, k))$ is required. It can be located on the top, left or back output pad.

- Assume that $f(V(i, j, k))$ is located on top side, which implies $f(V(i, j-1, k))$ is located on the bottom side. 
1. If $V(i, j-1, k)$ does not exist within the input pads, then we need to consider the case when $f(V(i, j-1, k))$ has a mismatch with bottom neighbor. Since we require this mismatch to cause three further errors in the neighborhood of $T(i, j, k)$, as argued above it requires an additional error checking function $g_{1}(f(V(i, j-1, k)))$ (that is input-sensitive to $\left.f(V(i, j-1, k))\right)$ to be located on at least one of the top, left, or back output pad.

2. If $V(i, j-1, k)$ exists in the input pads, then in case when $V(i, j-1, k)$ is mismatched, and three further errors in the neighborhood of $T(i, j, k)$ are required, it needs an additional error checking function $g_{1}^{\prime}(V(i, j-1, k))$ (that is input-sensitive to $V(i, j-1))$ to be located on at least one of the top, left or back output pad.

- Assume that $f(V(i, j, k))$ is located on left side, which implies $f(V(i-1, j, k))$ is located on the right side.

1. If $V(i-1, j, k)$ does not exist within the input pads, we need to consider the case when $f(V(i-1, j, k))$ is mismatched. Since three further errors are required, as argued above it requires an additional error checking function $g_{2}(f(V(i-1, j, k)))$ (that is input-sensitive to $\left.f(V(i-1, j, k))\right)$ to be located on at least one of the top, left or back output pad.

2. If $V(i-1, j, k)$ exists in the input pads, then in case when $V(i-1, j, k)$ is mismatched, and three further errors are required, it requires an additional error checking function $g_{2}^{\prime}(V(i-1, j, k)$ ) (that is input-sensitive to $V(i-1, j, k))$ to be present on at least one of the top, left or back output pads.

- Assume that $f(V(i, j, k))$ is located on the back side, which implies $f(V(i, j, k-1))$ is located on the front side.

1. If $V(i, j, k-1)$ does not exist within the input pads, we need to consider the case when $f(V(i, j, k-1))$ is mismatched. Since three further errors are required, as argued above it requires an additional error checking function $g_{3}(f(V(i, j, k-1)))$ (that is input-sensitive to $\left.f(V(i, j, k-1))\right)$ to be located on at least one of the top, left or back output pad.

2. If $V(i, j, k-1)$ exists in the input pads, then in case when $V(i, j, k-1)$ is mismatched, and three further errors are required, it requires an additional error checking function $g_{3}^{\prime}(V(i, j, k-1)$ ) (that is input-sensitive to $V(i, j, k-1))$ to be present on at least one of the top, left or back output pads.

Hence, an additional error checking pad $\left(g_{1}(f(V(i, j-1, k))), g_{1}^{\prime}(V(i, j-1, k))\right.$, $g_{2}(f(V(i-1, j, k))), g_{2}^{\prime}(V(i-1, j, k)), g_{3}(f(V(i, j, k-1)))$, or $\left.g_{3}^{\prime}(V(i, j, k-1))\right)$ is required on at least one of the output pads. Arguing in the same manner as above it can concluded that this cycle will keep on repeating. Hence, it is not possible to construct tile with a bounded number of parameters in the pads and we conclude that redundancy based compact error resilient schemes can not reduce error from $\epsilon$ to $\epsilon^{4}$. 


\section{Self-Healing Tile Set for Three Dimensional Assembly}

Winfree [31] provided the basis for studying self-healing in the self-assembly in a rigorous manner. We need to consider the repairability of a self-assembled structure in the face of a damage. A tile set is called self-healing, if at any point during errorfree growth, when $\mathrm{n}$ tiles are removed, subsequent error free growth will repair the damage rapidly [31]. Winfree's scheme of correctly repairing the damage (hole) is by ensuring that the holes are filled in the original forward direction of the algorithmic assembly and there is no backward growth in the holes.

Winfree proposed constructions of self-healing tile sets for two dimensional algorithmic self-assembly by replacing a single tile by a $3 \times 3$ (for simple assemblies like Sierpinski triangles), $5 \times 5$ (for general assemblies) and $7 \times 7$ (for additional robustness to nucleation errors) block. We have extended his constructions to three dimensions. Each three-dimensional tile is replaced by a $3 \times 3 \times 3$ block of threedimensional tiles to convert a tile set for simple assemblies into a self-healing tile set.

Figure 7 shows a $3 \times 3 \times 3$ block of tiles that replaces a computational tile. The internal glues inside the block are all unique to that block. The tile set given in Fig. 7 guarantees that if a complete block needs to regrow, then it has to start from frontmost, bottommost and rightmost corner. The tile to be placed at this corner is uniquely and correctly determined, by the assembled neighboring blocks. The corner tiles that have at least one output side facing towards another block should be assembled in the end after the assembly of all other tiles in the block, so that they can be determined uniquely from the inputs and not ambiguously from the outputs. We omit the figures of blocks showing the frame tile and seed tile, but they can be derived easily following the same logic. Similarly, Winfree's other constructions for self-healing in two-dimensions can also be extended to three dimensions to improve the self-healing tile set.

\section{Discussion}

In this paper, we presented a theoretical analysis of redundancy based compact error resilient tiling in two and three dimensions. We conjecture the following stronger results for three-dimensional assemblies that are currently open questions to be proved or disproved. We state our conjectures as follows:

Conjecture 2 For arbitrary Boolean functions $f_{1}, f_{2}$, and $f_{3}$, there exists no redundancy based compact error correction scheme that will reduce error from $\epsilon$ to $\epsilon^{3}$ in three-dimensional self-assembly.

Conjecture 3 For any functions $f_{1}, f_{2}$, and $f_{3}$ that are outside the restricted class of the functions defined in Theorem 6 there exists no redundancy based compact error correction scheme that will reduce error from $\epsilon$ to $\epsilon^{3}$ in three-dimensional selfassembly. 

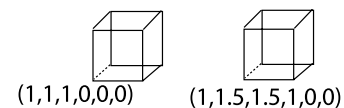

$(1,1.5,1.5,1,0,0)$
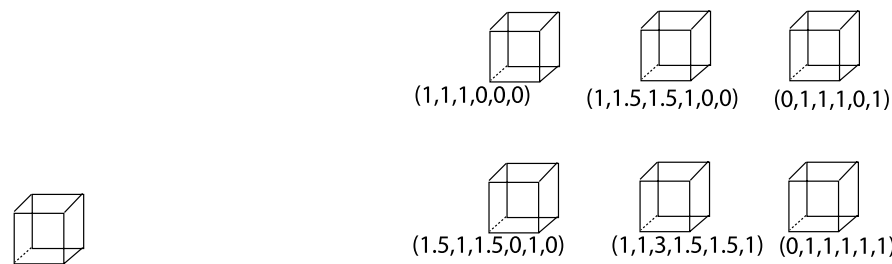

$(1.5,1,1.5,0,1,0)$

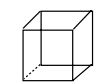

$(1,1,3,1.5,1.5,1) \quad(0,1,1,1,1,1)$

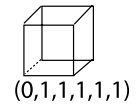

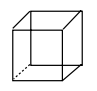

$(1,0,1,1,1,1) \quad(1,0,1,1,1,1)$

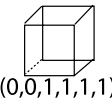

Back

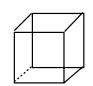

$(1.5,1.5,1,0,0,1)$

\section{.}
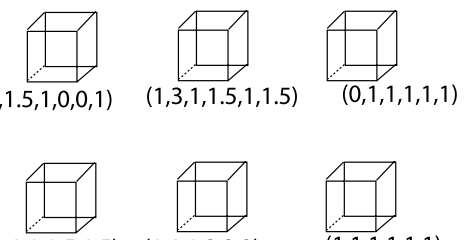

$(3,1,1,1,1.5,1.5)$
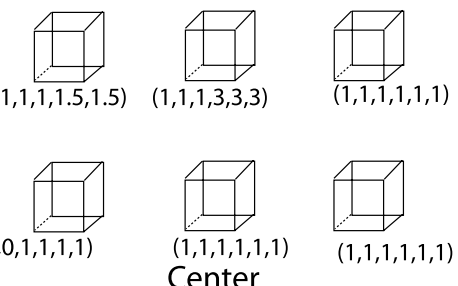

$(1,1,1,1,1,1)$
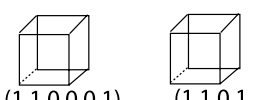

$(1,1,0,1,1,1)$
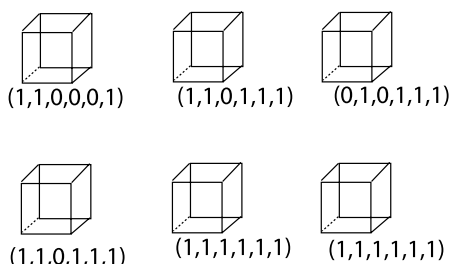

$(1,1,1,1,1,1)$

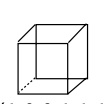

$(1,0,0,1,1,1)$

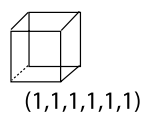

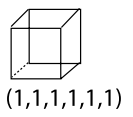

Front

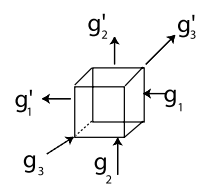

$\left(\mathrm{g}_{1}, \mathrm{~g}_{2^{\prime}}, \mathrm{g}_{3^{\prime}}, \mathrm{g}_{1}^{\prime}, \mathrm{g}_{2^{\prime}}^{\prime} \mathrm{g}_{3}^{\prime}\right)$

Fig. 7 Self-healing tile set for three dimensional assembly showing only a computational tile. One tile is replaced by a $3 \times 3 \times 3$ block of tiles. The 6-tuple shown below every tile shows the glue-strengths for its sides. The order of the glue strengths in the tuple is as shown in the single tile in the top left portion of the figure. The tuple $\left\langle g_{1}, g_{2}, g_{3}, g_{1}^{\prime}, g_{2}^{\prime}, g_{3}^{\prime}\right\rangle$ denotes the glue strength of $g_{1}$ on right, $g_{1}^{\prime}$ on left, $g_{2}$ on bottom, $g_{2}^{\prime}$ on top, $g_{3}$ on front and $g_{3}^{\prime}$ on the back side of the tile. This construction corresponds to a computational tile in the assembly, which has the glue strength 1 on each of its 6 faces

Conjecture 4 For any Boolean functions $f_{1}, f_{2}$, and $f_{3}$, there exists no redundancy based compact error resilient scheme that can reduce error from $\epsilon$ to $\epsilon^{4}$ in threedimensional self-assembly. 
The immediate future work will be to prove or disprove these conjectures. We have presented a three-dimensional extension to Winfree's self-healing tile set in twodimensions. It remains an open question if it is possible to design a compact selfhealing tile set for two and three-dimensional self-assembly.

Acknowledgement The work is supported by NSF EMT Grants CCF-0523555 and CCF-0432038.

\section{References}

1. Aviram, A., Ratner, M.: Molecular Electronics: Science and Technology. New York Academy of Sciences, New York (1998)

2. Bondarenko, B.A.: Generalized Pascal Triangles and Pyramids, Their Fractals, Graphs and Applications. The Fibonacci Association (1993). Translated from Russian and edited by R.C. Bollinger

3. Bowden, N., Terfort, A., Carbeck, J., Whitesides, G.M.: Self-assembly of mesoscale objects into ordered two-dimensional arrays. Science 276(11), 233-235 (1997)

4. Bumm, L.A., Arnold, J.J., Charles, L.F., Dunbar, T.D., Allara, D.L., Weiss, P.S.: Directed selfassembly to create molecular terraces with molecularly sharp boundaries in organic monolayers. J. Am. Chem. Soc. 121, 8017-8021 (1999)

5. Chelyapov, N., Brun, Y., Gopalkrishnan, M., Reishus, D., Shaw, B., Adleman, L.: DNA triangles and self-assembled hexagonal tilings. J. Am. Chem. Soc. 126, 13924-13925 (2004)

6. Chen, H.L., Goel, A.: Error free self-assembly using error prone tiles. In: DNA Based Computers 10, pp. 274-283 (2004)

7. Chen, H.L., Cheng, Q., Goel, A., Huang, M.D., de Espanes, P.M.: Invadable self-assembly: Combining robustness with efficiency. In: Proceedings of the 15th Annual ACM-SIAM Symposium on Discrete Algorithms (SODA), pp. 890-899 (2004)

8. Chen, J., Reed, M.A., Rawlett, A.M., Tour, J.M.: Large on-off ratios and negative differential resistance in a molecular electronic device. Science 286, 1550-1552 (1999)

9. Clark, T.D., Ferrigno, R., Tien, J., Paul, K.E., Whitesides, G.M.: Template-directed self-assembly of 10-microm-sized hexagonal plates. J. Am. Chem. Soc. 124(19), 5419-5426 (2002)

10. Jonoska, N., Karl, S.A., Saito, M.: Three dimensional DNA structures in computing. BioSystems 52, 143-153 (1999)

11. LaBean, T.H., Yan, H., Kopatsch, J., Liu, F., Winfree, E., Reif, J.H., Seeman, N.C.: The construction, analysis, ligation and self-assembly of DNA triple crossover complexes. J. Am. Chem. Soc. 122, $1848-1860$ (2000)

12. Lagoudakis, M.G., LaBean, T.H.: 2-D DNA self-assembly for satisfiability. In: DNA Based Computers V. DIMACS, vol. 54, pp. 141-154. American Mathematical Society, Providence (2000)

13. Liu, D., Wang, M., Deng, Z., Walulu, R., Mao, C.: Tensegrity: Construction of rigid DNA triangles with flexible four-arm DNA junctions. J. Am. Chem. Soc. 126, 2324-2325 (2004)

14. Mao, C., Sun, W., Seeman, N.C.: Designed two-dimensional DNA Holliday junction arrays visualized by atomic force microscopy. J. Am. Chem. Soc. 121, 5437-5443 (1999)

15. Martin, B.R., Furnange, D.C., Jackson, T.N., Mallouk, T.E., Mayer, T.S.: Self-alignment of patterned wafers using capillary forces at a water-air interface. Adv. Funct. Mater. 11, 381-386 (2001)

16. Paukstelis, P.J., Nowakowski, J., Birktoft, J.J., Seeman, N.C.: Crystal structure of a continuous threedimensional DNA lattice. Chem. Biol. 11, 1119-1126 (2004)

17. Petty, M.C., Bryce, M.R., Bloor, D.: An introduction to Molecular Electronics. Oxford University Press, New York (1995)

18. Reed, M.A., Zhou, C., Muller, C.J., Burgin, T.P., Tour, J.M.: Conductance of a molecular junction. Science 278, 252-254 (1997)

19. Reif, J.H.: Local parallel biomolecular computation. In: Rubin, H., Wood, D.H. (eds.) DNA-Based Computers 3. DIMACS, vol. 48, pp. 217-254. American Mathematical Society, Providence (1999)

20. Reif, J.H., Sahu, S., Yin, P.: Compact error-resilient computational DNA tilings. Nanotechnol. Sci. Comput. 79-103 (2006)

21. Rothemund, P.W.K.: Using lateral capillary forces to compute by self-assembly. Proc. Natl. Acad. Sci. USA 97(3), 984-989 (2000)

22. Sahu, S., Reif, J.H.: Capabilities and limits of compact error resilience methods for algorithmic selfassembly in two and three dimensions. In: LNCS, vol. 4287, pp. 223-238 (2006) 
23. Schulman, R., Winfree, E.: Programmable control of nucleation for algorithmic self-assembly. In: LNCS, vol 3384, pp. 319-328 (2005)

24. Seeman, N.C.: DNA in a material world. Nature 421, 427-431 (2003)

25. Soloveichik, D., Winfree, E.: Complexity of self-assembled shapes. SIAM J. Comput. 36, 1544-1569 (2007)

26. Wang, H.: Proving theorems by pattern recognition ii. Bell Syst. Tech. J. 40, 1-41 (1961)

27. Waybright, S.M., Singleton, C.P., Tour, J.M., Murphy, C.J., Bunz, U.H.F.: Synthesis and self-assembly of an oligonucleotide-modified cyclobutadiene complex. Organometallics 19, 368-370 (2000)

28. Whitesides, G.M., Grzybowski, B.: Self-assembly at all scales. Science 295, 2418-2421 (2002)

29. Winfree, E.: Complexity of restricted and unrestricted models of molecular computation. In: Lipton, R.J., Baum, E.B. (eds.) DNA Based Computers 1. DIMACS, vol. 27, pp. 187-198. American Mathematical Society, Providence (1996)

30. Winfree, E.: Simulation of computing by self-assembly. Technical Report 1998.22, Caltech (1998)

31. Winfree, E.: Self-healing tile sets. Nanotechnol. Sci. Comput. 55-78 (2006)

32. Winfree, E., Bekbolatov, R.: Proofreading tile sets: Error correction for algorithmic self-assembly. In: DNA Based Computers 9. LNCS, vol. 2943, pp. 126-144. Springer, Berlin (2004)

33. Winfree, E., Liu, F., Wenzler, L.A., Seeman, N.C.: Design and self-assembly of two-dimensional DNA crystals. Nature 394(6693), 539-544 (1998)

34. Winfree, E., Yang, X., Seeman, N.C.: Universal computation via self-assembly of DNA: Some theory and experiments. In: Landweber, L.F., Baum, E.B. (eds.) DNA Based Computers II. DIMACS, vol. 44, pp. 191-213. American Mathematical Society, Providence (1999)

35. Xiong, X., Hanein, Y., Fang, J., Wang, Y., Wang, W., Schwartz, D., Bohringer, K.: Controlled multibatch self-assembly of microdevices. J. Microelectromech. Syst. 12, 117-127 (2003)

36. Yan, H., Feng, L., LaBean, T.H., Reif, J.H.: Parallel molecular computation of pair-wise XOR using DNA string tile. J. Am. Chem. Soc. 125(47) (2003)

37. Yan, H., LaBean, T.H., Feng, L., Reif, J.H.: Directed nucleation assembly of DNA tile complexes for barcode patterned DNA lattices. Proc. Natl. Acad. Sci. USA 100(14), 8103-8108 (2003)

38. Yan, H., Park, S.H., Finkelstein, G., Reif, J.H., LaBean, T.H.: DNA-templated self-assembly of protein arrays and highly conductive nanowires. Science 301(5641), 1882-1884 (2003)

39. Zhou, C.: Atomic and molecular wires. PhD thesis, Yale University (1999) 